\title{
Adaptive Repetitive Control with Feedforward Scheme for Grid-Connected Inverters
}

\author{
Mohammad Abusara ${ }^{1}$, Suleiman Sharkh ${ }^{2}$ and Pericle Zanchetta ${ }^{3}$
}

\begin{abstract}
${ }^{1}$ Renewable Energy Research Group, College of Engineering, Mathematics and Physical Sciences, University of Exeter, Penryn Campus, TR10 9EZ. T: 01326371885. E: M.Abusara@exeter.ac.uk.

${ }^{2}$ Electro-Mechanical Engineering Research Group, Engineering Sciences, University of Southampton, SO17 1BJ. T: 02380593397, E: suleiman@soton.ac.uk.

${ }^{3}$ Electronic Engineering, University of Nottingham, NG7 2RD. T: 0115 9515592, E: Pericle.Zanchetta@nottingham.ac.uk.
\end{abstract}

Abstract - This paper is concerned with control of grid connected inverters using odd harmonic repetitive scheme. Due to the inherent long convergence period of repetitive control, a feedforward loop of the fundamental component of the grid voltage is used to make sure that the inverter's output current is bounded during the convergence period. The well know deficiency of repetitive control in rejecting harmonics when used with varying grid frequency has been solved by using an adaptive scheme in which the switching and sampling frequencies vary with respect to the grid frequency. The main qualities of the proposed controller are its effectiveness, simplicity and straightforward of implementation using a Digital Signal Processor. Simulation and practical results are presented to demonstrate the controller capabilities.

Keywords-grid-connected inverters; repetitive control; adaptive frequency control.

NOMENCLATURE

$n \quad$ Number of samples on one fundamental cycle

$N_{\text {сри }} \quad$ PWM counter period

$N_{\text {cpu_o }} \quad$ Nominal PWM counter period

$T_{\text {сри }}, f_{\text {сри }}$ DSP clock period and frequency

$T_{g}, f_{g} \quad$ Grid voltage fundamental period and frequecny

$T_{i n v}, f_{i n v}$ inverter modulating signal period and frequency

$T_{d} \quad$ DSP computational time delay

$T_{s}, f_{s} \quad$ Sampling period and frequency

$T_{\text {so }}, f_{\text {so }}$ Nominal sampling period and frequency

$T_{s w}, f_{s w} \quad$ Switching period and frequency

\section{INTRODUCTION}

In grid-connected inverters repetitive controllers (RC) [RC references] have the advantage of much higher gain at the fundamental frequency and its harmonic than that provided by classical PID controllers and their derivatives [PID references]. Hence, they are capable of producing significantly higher quality output current, as demanded by national and international standards [harmonic 
standards references], over a wider power output range than classical PID. They are also much simpler to implement in practice than proportional resonance controllers [PR references].

However, using RC alone in grid-connected inverters may not provide satisfactory transient response. The conventional RC structure [27]-[29], which has the ability to reject both even and odd harmonics has an inherent control delay of one fundamental period and thus suffers from slow dynamic response. Odd-harmonic RC [18]-[20], [30],[31], which makes use of the fact that most AC systems have only odd harmonics in their spectrum, has an inherent time delay of half the period of a fundamental cycle, which makes its dynamic response faster than that of conventional RC. But that may not be sufficient to deal with disturbance caused by grid voltage swell or sag could result in the inverter generating output current that is much higher than the reference current. Relying on $\mathrm{RC}$ alone to deal with such disturbance means that the current error could be too high during the RC convergence time, which could cause an over current fault.

Another shortcoming of $\mathrm{RC}$ is that its gain falls very rapidly either side of its resonant frequencies. Hence, if the grid frequency deviates slightly from its nominal value, the high resonant gains will not be lined up with the grid fundamental frequency and its harmonics causing serious deterioration in the controller's performance. Hornik and Zhong [24],[25] proposed voltage control of grid connected inverters with a frequency adaptive mechanism that is based on Hळ repetitive control. The internal model of the system consists of a delay unit $e^{-T_{D} s}$ cascaded with a low-pass filter, $W(s)=\omega_{c} / s+\omega_{c}$. The adaptive mechanism is based on varying the cut-off frequency of the filter $\omega_{c}$ according to the varying grid frequency. According to the authors, this mechanism is effective for a grid frequency variation of only $\pm 0.2 \mathrm{~Hz}$. If the grid variation is higher than this limit, the controller delay $T_{D}$ needs to be changed. However, for low sampling frequency, an adaptive delay is impossible to be implemented without further deterioration of the controller performance [24].

To overcome the above deficiencies, this paper proposes an odd-harmonic repetitive controller, with a feed forward loop to speed its transient response and a fast adaptive frequency mechanism. The frequency adaptive feature is based on varying both switching and sampling frequencies according to the grid frequency and thus keeping the number of delay samples of the RC constant. The design results in fast convergence to steady state, within one fundamental cycle. The novelty of the paper is twofold: improving the transient response of the RC by using a feedforward loop of the grid voltage and improving the steady state performance of the RC by using an adaptive scheme that tracks the variation in grid frequency. The main qualities of the proposed controller are its effectiveness, simplicity and straightforward of implementation using a Digital Signal Processor.

The rest of the paper is organized as follows: section II describes the controller structure. Section III describes the proposed frequency adaptive mechanism. Finally, simulation and practical results are presented in sections IV and V, respectively. 


\section{CONTROLler Structure AND Design}

Fig. 1a shows the circuit diagram of a conventional 2-level grid-connected inverter with $L C L$ filter [3],[7]. The inverter parameters used in this paper are listed in Table I. The selection of the filter component values is a trade-off between inductor size, IGBT switching losses, resonant frequency, and the degree of filter attenuation at the switching frequency. The switching frequency has been chosen to be $8 \mathrm{kHz}$ which is limited by the maximum IGBTs power dissipation. The $L_{1}$ inductance of $350 \mu \mathrm{H}$ has been chosen to limit the ripple current to a value of $~ 25 \%$ of the rated current (140A) as dictated by the ratings and losses in the IGBT modules. The values of $L_{2}$ and $C$ have been chosen so the impedance of $L_{2}$ at the switching frequency is several times bigger than the impedance of $C$ in order to prevent the ripple current from being injected into the grid. The computational time delay is determined by the speed of the DSP in processing the real time calculations.

The block diagram that represents the control scheme implemented in each phase is shown in Fig. 1b. The controller consists of an outer loop of the output current and an inner loop of the capacitor current to provide active damping. This structure increases the degree of freedom in designing the controller compared with the widely used one-feedback loop of $L_{1}$ current because two controller gains $\left(K_{C}\right.$ and $K$ ) can be optimized instead of only one controller gain.

A plug-in repetitive controller transfer function $\left(G_{R C}\right)$ is implemented to reject current harmonics caused by grid voltage harmonics. The controller diagram is simplified as shown in Fig. 1c where $D_{g}(z)$ represents the grid disturbance and $G_{p}(z)$ is the open loop transfer function in the discrete time domain that takes into account the zero order hold and time delay effects and is given as

$$
G_{p}(z)=\frac{Z\left(\frac{1-e^{-s T_{s}}}{s} e^{-s T_{d}} \frac{1}{L_{1} L_{2} C s^{3}+\left(L_{1}+L_{2}\right) s}\right)}{1+Z\left(\frac{1-e^{-s T_{s}}}{s} e^{-s T_{d}} \frac{K_{C} L_{2} C s^{2}}{L_{1} L_{2} C s^{3}+\left(L_{1}+L_{2}\right) s}\right)},
$$

where $\mathrm{Z}$ is the $\mathrm{z}$-transform operator, $T_{s}$ is the sampling time, $T_{d}$ is the computational time delay, and $K_{C}$ is the capacitor current loop gain. The transfer function of the odd harmonic repetitive controller is given in [30] as

$$
G_{R C}(z)=-\frac{K_{R} z^{m} Q(z) z^{-n / 2}}{1+Q(z) z^{-n / 2}},
$$

where $Q(z)$ is a low pass filter, $K_{R}$ is the repetitive controller gain, $n$ is the number of samples in one fundamental cycle and $z^{m}$ is a non-causal phase lead unit.

The repetitive controller is capable of rejecting the disturbance of the fundamental harmonic and its multiples. However, the convergence time of the repetitive controller is relatively long and therefore a feedforward loop of the fundamental nominal component of the grid voltage is implemented to reduce the current error during the convergence time of the RC. 


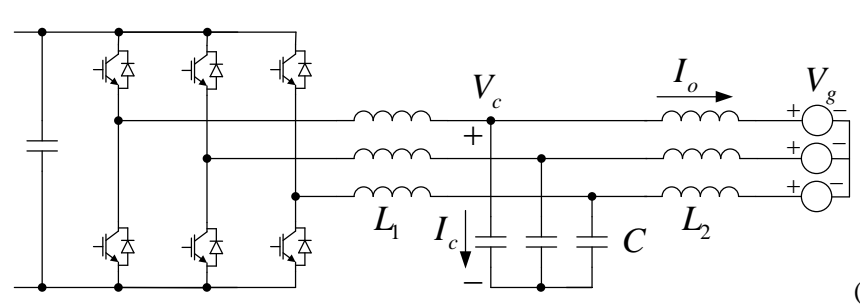

(a)

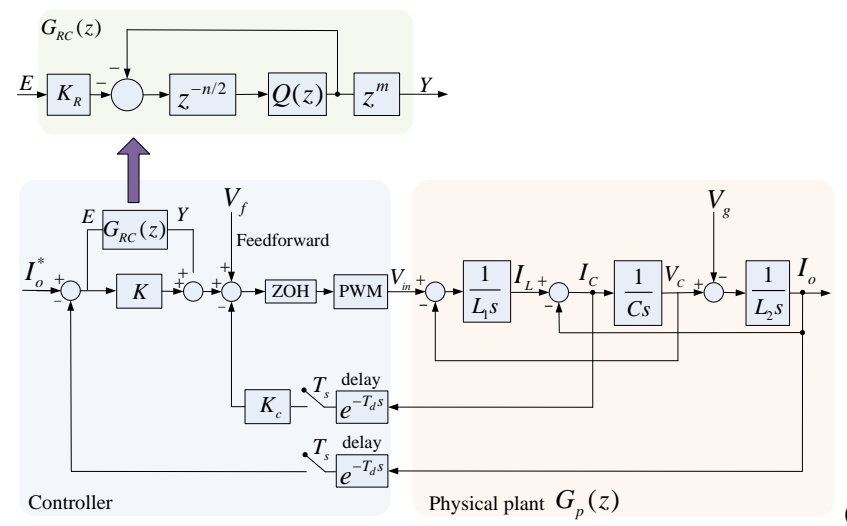

(b)

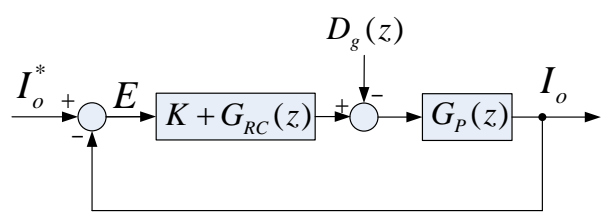

(c)

Fig. 1. Grid connected inverter (a) and its controller (b) and simplified controller (c)

TABLE I

INVERTER PARAMETERS

\begin{tabular}{l|c|c}
\hline \multicolumn{1}{c|}{ Description } & Symbol & Value \\
\hline Inverter side filter inductor & $L_{1}$ & $350 \mu \mathrm{H}$ \\
\hline Filter capacitor & $C$ & $80 \mu \mathrm{F}$ \\
\hline Grid side filter inductor & $L_{2}$ & $50 \mu \mathrm{H}$ \\
\hline Nominal grid voltage & $V_{g o}$ & $230 \mathrm{~V}(\mathrm{rms})$ \\
\hline Inverter dc voltage & $V_{d c}$ & $700 \mathrm{Vdc}$ \\
\hline Nominal grid frequency & $f_{g o}$ & $50 \mathrm{~Hz}$ \\
\hline Nominal switching frequency & $f_{s w o}$ & $8 \mathrm{kHz}$ \\
\hline Nominal sampling frequency & $T_{s o}$ & $16 \mathrm{kHz}$ \\
\hline Computational time delay & $T_{d}$ & $10 \mu \mathrm{s}$ \\
\hline
\end{tabular}

The controller design involves the determination of $K_{C} K, Q(z), K_{R}$, and $m$. The error signal $E$ (see Fig. 1c) can be expressed as

$$
E(z)=\frac{I_{o}^{*}(z)+D_{g}(z) G_{p}(z)}{1+K G_{p}(z)+G_{p}(z) G_{R C}(z)} .
$$

Substituting (2) in (3) and rearranging gives 


$$
\frac{E(z)}{I_{o}^{*}(z)+D_{g}(z) G_{p}(z)}=\frac{\left(1+z^{-n / 2} Q(z)\right)}{\left(1+K G_{p}(z)\right)\left(1-z^{-n / 2} R(z)\right)},
$$

where

$$
R(z)=Q(z)\left(\frac{K_{R} z^{m} G_{p}(z)}{1+K G_{p}(z)}-1\right)
$$

Equation (4) can be represented by the block diagram in Fig. 2 which consists of three cascaded transfer functions: $T_{1}(z), T_{2}(z)$, and $T_{3}(\mathrm{z}) . T_{1}(\mathrm{z})$ is the closed loop transfer function without the repetitive controller and its stability can be guaranteed by choosing $K_{C}$ and $K$. The stability of the second transfer function $T_{2}(z)$ can be guaranteed by choosing a stable low pass filter $Q(z)$. The stability of the third transfer function $T_{3}(z)$ which contains a positive feedback loop can be guaranteed using the small gain theorem, i.e., the error signal will be bounded if the magnitude of the open loop transfer function is less than 1 for all values of frequencies. Hence

$$
\|R(z)\|_{\infty}<1
$$

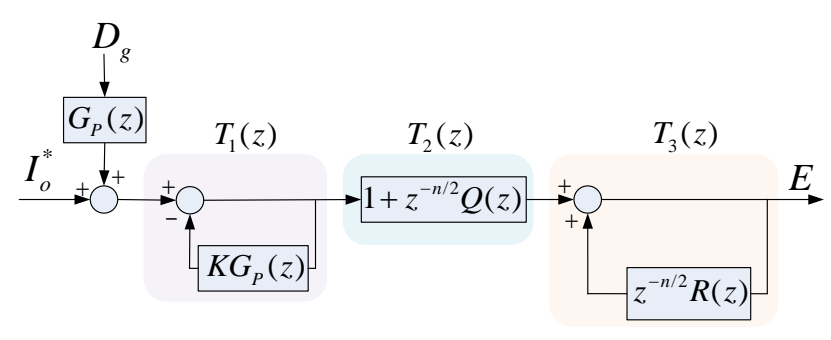

Fig. 2. Block diagram of the system error

\section{A. Selection of $K_{C}$ and $K$}

The selection of the capacitor current loop gain $K_{C}$ and the output current loop gain $K$ is determined to give good stability margins. By analysing $G_{p}(z)$ in Matlab SISO Design Tool, the gains $K_{C}$ and $K$ have been set to 5 and 3, respectively. This selection gives gain margin of $5.6 \mathrm{~dB}$, phase margin of $51 \mathrm{deg}$, damping ratio $\zeta=0.33$, and settling time $t_{\mathrm{s}}=0.5 \mathrm{~ms}$.

\section{B. Selection of $Q(z)$}

$Q(z)$ is a zero-phase low pass filter that is used to attenuate the high frequency resonant peaks of the RC, to ensure stability. It has the following structure [32].

$$
Q(z)=\alpha_{1} z+\alpha_{0}+\alpha_{1} z^{-1}
$$

where, $\alpha_{o}$ and $\alpha_{1}$ are set to 0.5 , and 0.25 , respectively [32]. 
The bode diagram of the open loop transfer function $\left(K+G_{R C}(z)\right) G_{p}(z)$ is shown in Fig. 3; it confirms the system's stability as the high gains near the crossover frequency have been attenuated and the system has positive stability margins.

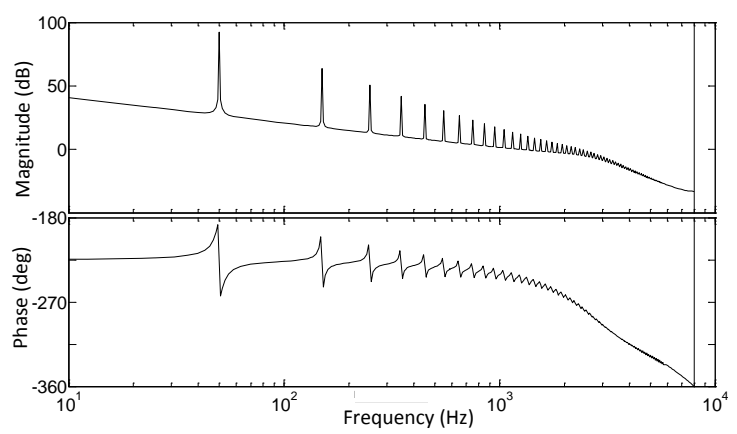

Fig. 3. Bode diagram of the open loop transfer function

$$
\left(K+G_{R C}(z)\right) G_{p}(z) \text {, with } Q(z)=0.25 z+0.5+0.25 z^{-1}, m=1, K_{R}=0.5 \quad(\mathrm{GM}=5.0 \mathrm{~dB}, \mathrm{PM}=44.1 \mathrm{deg})
$$

\section{Selection of $K_{R}$ and $m$}

The value of the RC gain $K_{R}$ needs to be carefully selected as it is a key parameter for error convergence and system stability. A high repetitive controller gain $K_{R}$ results in fast error convergence but the feedback system becomes less stable. The non-causal phase lead unit of $m$ is normally used to compensate for any delay or phase lag introduced by the physical plant and controller transfer function. Implementing $z^{m}$ is not possible practically unless it is cascaded with the delay units of the RC. The design criterion used in this paper is maximizing $K_{R}$ so as to reduce the RC convergence time but at the same time minimizing $\|R(z)\|_{\infty}$ so as to increase the stability margins. By plotting $\|R(z)\|_{\infty}$ versus $K_{R}$ and $m$ it can be shown that when $m=0$ the system is only stable when $K_{R}<0.6\left(\|R(z)\|_{\infty}=0.99\right.$ when $\left.K_{R}=0.6\right)$. Increasing $m$ does improve stability and the best value is $m=3$ as this corresponds to the lowest possible $\|R(z)\|_{\infty}$. The minimum value for $\|R(z)\|_{\infty}$ occurs when $K_{R}=2.8$ and $m=3$ and hence they have been chosen for this design.

\section{FREQUENCY ADAPTIVE REPETITIVE CONTROL}

In order to keep the RC effective, it has to adapt to the varying grid frequency. The performance of the repetitive controller is not guaranteed unless the high resonant gains line up with the grid harmonics. Therefore, the time delay of the repetitive controller needs to adapt to the changes in grid voltage fundamental period. The number of delay samples $n$ can be changed to cope with the changing frequency. However, unless the switching frequency (and hence the sampling frequency) are very high with respect to the fundamental frequency, this mechanism will not result in precise control of the time delay used by the repetitive controller. For example, if the sampling frequency is $4 \mathrm{kHz}$, for a fundamental frequency of $50 \mathrm{~Hz}$, the number of samples per cycle is $4000 / 50=80$ samples. Each sample is equivalent to $0.253 \mathrm{~ms}$ which means that the minimum change in grid 
frequency that this scheme can deal with is approximately $0.63 \mathrm{~Hz}$. If the grid frequency deviates by only $0.15 \mathrm{~Hz}$ from its nominal value, the RC gain at the $5^{\text {th }}$ harmonic can reduce by about $20 \%$ of its nominal value. For $0.60 \mathrm{~Hz}$ deviation, the RC becomes completely ineffective. Therefore, varying the number of samples will not provide good tracking of grid frequency without deteriorating the RC performance.

The mechanism proposed in this paper is to change the switching frequency and the sampling frequency such that the ratio of the sampling frequency to the fundamental frequency remains constant and hence $n$ does not need to change. The mechanism benefits form the high precision of the DSP clock used to implement the controller. Fig. 4 shows how the PWM carrier is implemented in the DSP. In this paper, the sampling frequency $f_{s}$ is set to be twice the switching frequency such as $f_{s}=2 f_{s w}$. A counter that is based on the Central Processing Unit (CPU) clock is set to count up and down periodically. The PWM counter period $N_{c p u}$ is set to determine the required sampling period. Hence

$$
N_{c p u}=\frac{T_{s}}{T_{c p u}},
$$

where $T_{s}$ and $T_{\text {срu }}$ are the sampling and CPU clock periods, respectively. The number of samples $n$ per one fundamental cycle is given by,

$$
n=\frac{T_{g}}{T_{s}},
$$

where $T_{g}$ is the grid voltage fundamental period. The inverter modulating signal period is given by

$$
T_{i n v}=n T_{s} .
$$

Substituting (8) into (10) gives

$$
T_{i n v}=n N_{c p u} T_{\text {cpu }} .
$$

According to (11), in order to vary the inverter's modulating signal period $T_{i n v}$ and hence the frequency $f_{\text {inv }}$ while maintaining $n$ constant, the number of counts $N_{c p u}$ needs to be changed.

Fig. 5 shows the proposed controller of the PWM counter period $N_{c p u}$. The grid voltage fundamental period $T_{g}$ is sensed (by a PLL or zero crossing detector) and divided by $n T_{c p u}$ to calculate the demand PWM counter period $N_{c p u}^{*}$. The period error $E_{N}$ is fed into a Proportional-Integrator (PI) controller to calculate $\Delta N_{c p u}$ which is added to the nominal PWM counter period $N_{c p u_{\_} o}$ to produce $N_{c p u}$. The nominal PWM counter period $N_{c p u_{\_} o}$ is calculated as

$$
N_{c p u_{-} o}=T_{\text {so }} / T_{c p u} \text {, }
$$

where $T_{\text {so }}$ is the nominal switching period. 
To highlight the advantage of this mechanism over changing $n$, consider the case where the CPU frequency $f_{c p u}=150 \mathrm{MHz}, n=320$, the nominal switching frequency $f_{\text {so }}=16 \mathrm{kHz}$, according to (12) the nominal PWM counter period $N_{\text {cpц_o }}=150 \mathrm{MHz} / 16 \mathrm{kHz}=9375$. If the inverter modulating signal frequency $f_{\text {iiv }}$ is controlled by varying $n$ then by reducing $n$ by 1 , $f_{\text {inv }}$ will be given by

$$
f_{\text {inv }}=\frac{150 \mathrm{MHz}}{(320-1) * 9375}=50.1567 \mathrm{~Hz} .
$$

However, if $f_{\text {inv }}$ is controlled by varying $N_{c p u}$ as proposed here, by reducing $N_{c p u}$ by 1 count, then $f_{\text {inv }}$ will be given by

$$
f_{\text {inv }}=\frac{150 \mathrm{MHz}}{320 *(9375-1)}=50.0053 \mathrm{~Hz} \text {. }
$$

It can be noticed that varying $N_{c p u}$ gives 29 times more precision in controlling the frequency.

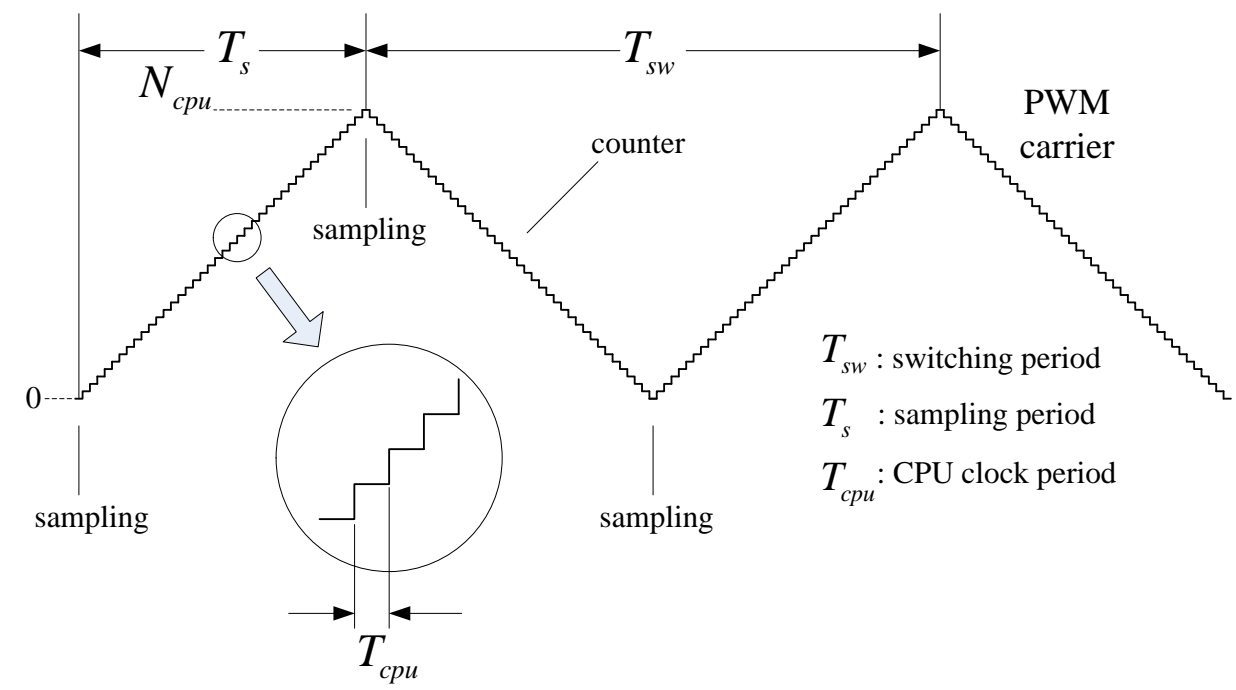

Fig. 4. PWM implementation in the DSP

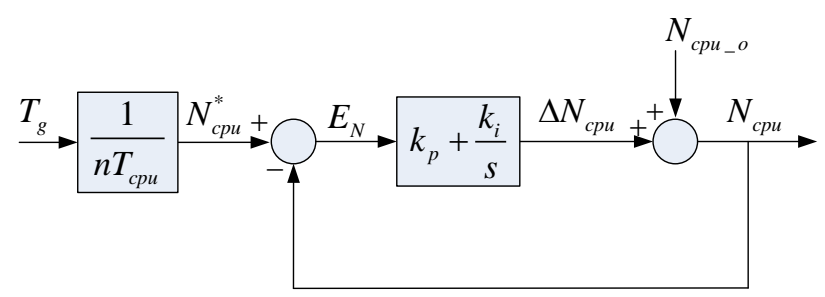

Fig. 5. PWM period control

\section{A. Frequency controller design}

According to most of the grid codes of practice, the grid frequency variation has a maximum slope of $1 \mathrm{~Hz} / \mathrm{sec}$ [12]. This is nearly equivalent to increasing or decreasing the grid period by about $0.4 \mathrm{~ms}$ per second. The design objective is to track this 
variation and maintain the error signal between the PWM counter period $N_{c p u}$ and its demand $N_{c p u}^{*}$ to the minimum of one count. The deviation in grid frequency and hence the PWM counter period demand $N_{c p u}^{*}$ will be modeled as a ramp function as:

$$
N_{c p u}^{*}=\frac{D}{s^{2}},
$$

where $D$ is the rate of change of $N_{c p u}^{*}$ in count/sec. From Fig. 5 the error $E_{N}$ (error signal between the PWM counter period $N_{c p u}$ and its demand $N_{c p u}^{*}$ ) is given by

$$
E_{N}(s)=\frac{N_{c p u}^{*}-N_{c p u_{-} o}}{1+\left(k_{p}+\frac{k_{i}}{s}\right)} .
$$

The steady state error $E_{N s s}$ for a ramp input of $N_{c p u}^{*}$ can be calculated by substituting (15) in (16) and using the final-value theorem,

$$
E_{\mathrm{Nss}}=\lim _{s \rightarrow 0}\left(s E_{N}\right)=\frac{D}{k_{i}} .
$$

Maximum $D$ is 184 count/sec (equivalent to $0.4 \mathrm{~ms}$ per second). The integral gain $k_{i}$ is chosen to give the minimum possible steady state frequency error which is one count. Therefore $k_{i}$ is set to 184 . The proportional gain $k_{p}$ is normally set to deal with transient response to a step input, In this case, a step change in grid frequency is unlikely and the proportional gain is set to 10.

\section{Simulation Results}

Detailed simulation model of the 3-phase inverter presented in Fig. 1 has been built using the MATLAB SimPowerSystems. The system parameters were listed in Table I and the controller parameters are listed in Table II. Grid voltage harmonics were measured in the laboratory and similar values were included in the simulation model. Table III lists the amplitudes of the main grid harmonics. The total grid voltage THD was measured to be $1.9 \%$.

Fig. 6a shows the output current without RC for a 14A (rms) demand. The THD is $14.2 \%$. Fig. 6 b shows the steady state output current with RC. The current THD is reduced to only $0.8 \%$. The effectiveness of RC in improving the current THD is clearly noticed. Fig. 6c shows the output current harmonics with and without the RC.

Fig. 7a shows the output current during starting when the feedforward is deactivated. It can be noticed that during the first half cycle the output current is nearly five times the reference current. This is due to the disturbance caused by the fundamental component of the grid voltage. Fig. 7b shows the output current when the feedforward of the nominal fundamental component of the grid voltage is activated. The error in the first half cycle is small and it is due to the disturbance caused by the grid higher 
harmonics. In Fig 7c, an error of 35V is suddenly introduced in the feedforward loop. This causes immediate change in the output current. The RC corrects for this error but it takes half fundamental cycle to converge. This shows the importance of the feedforward loop in complementing the RC.

To test the effectiveness of the adaptive frequency control, the grid frequency is set to start to change from $50 \mathrm{~Hz}$ to $50.2 \mathrm{~Hz}$ at simulation time $\mathrm{t}=0.2 \mathrm{sec}$. The slope of change is $1 \mathrm{~Hz} / \mathrm{sec}$. Fig. 8 shows the output current THD with and without activating the frequency adaptive control. Without the adaptive mechanism, the current THD increases as the frequency deviation increases and it reached $10 \%$. Once the grid frequency reaches $50.2 \mathrm{~Hz}$ and stops deviating, the THD dropped to $4 \%$. The adaptive frequency control on the other hand was able to keep the output current THD at $0.3 \%$ all time.

TABLE II

CONTROLLER PARAMETERS

\begin{tabular}{l|c|c}
\hline \multicolumn{1}{c|}{ Description } & Symbol & Value \\
\hline CPU frequency & $f_{c p u}$ & $150 \mathrm{MHz}$ \\
\hline Nominal CPU timer period & $N_{c p u \_o}$ & 9375 \\
\hline Number of samples in one fundamental cycle & $n$ & 320 \\
\hline Output current feedback gain & $K$ & 3 \\
\hline Capacitor current feedback gain & $K_{C}$ & 5 \\
\hline RC gain & $K_{R}$ & 2.8 \\
\hline Noncausal phase lead & $m$ & 3 \\
\hline Frequency control proportional gain & $k_{p}$ & 10 \\
\hline Frequency control integral gain & $k_{i}$ & 184 \\
\hline Filter $Q(z)$ coefficient 1 & $\propto_{0}$ & 0.5 \\
\hline Filter $Q(z)$ coefficient 2 & $\propto_{1}$ & 0.25 \\
\hline
\end{tabular}

TABLE III

MAIN GRID HARMONICS

\begin{tabular}{l|l}
\hline Harmonic & \% of fundamental \\
\hline \hline 3 & 0.492 \\
\hline 5 & 0.923 \\
\hline 7 & 0.277 \\
\hline 9 & 0.369 \\
\hline 11 & 0.492 \\
\hline 13 & 0.338 \\
\hline 15 & 0.308 \\
\hline 17 & 0.185 \\
\hline
\end{tabular}

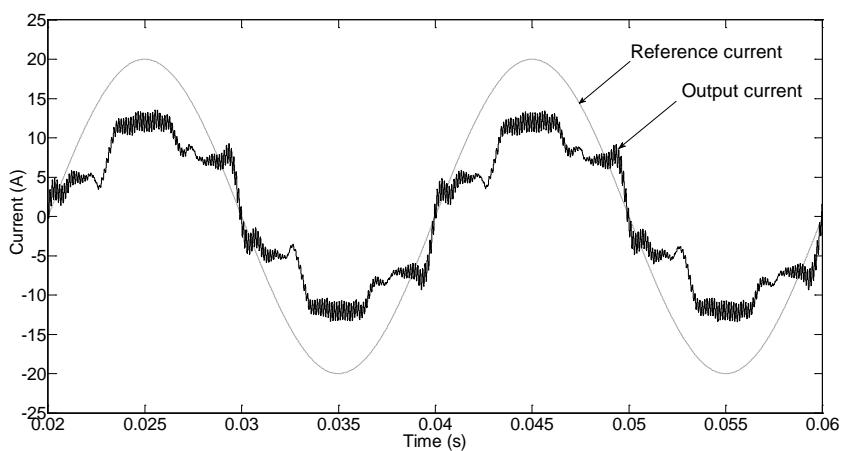


(a) Simulated steady state output current without RC (THD = 14.2\%)

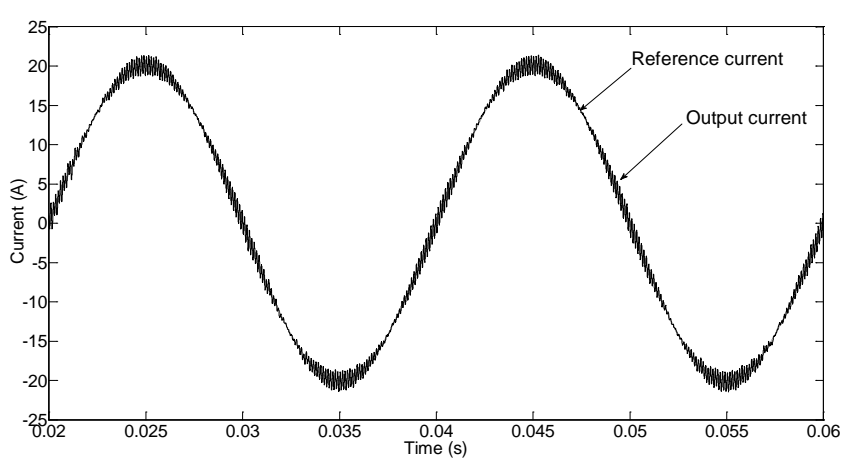

(b) Simulated steady state output current with RC (THD $=0.8 \%$ )

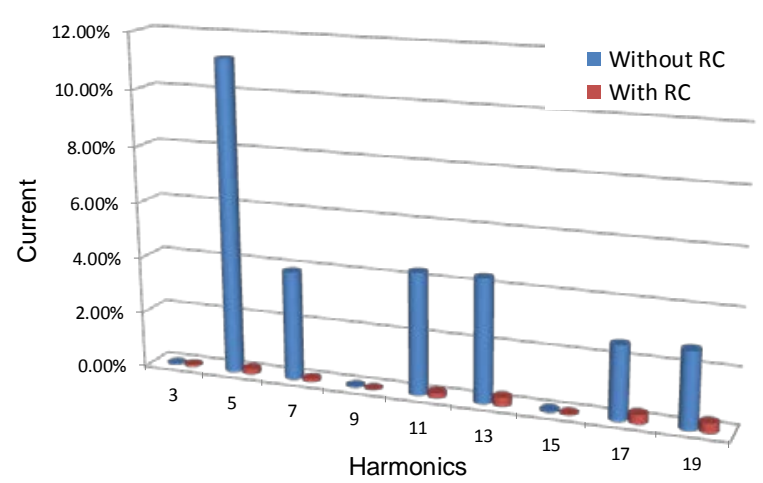

(c) Output current harmonics

Fig. 6. Simulated steady state output current and its harmonic spectrum with and without RC

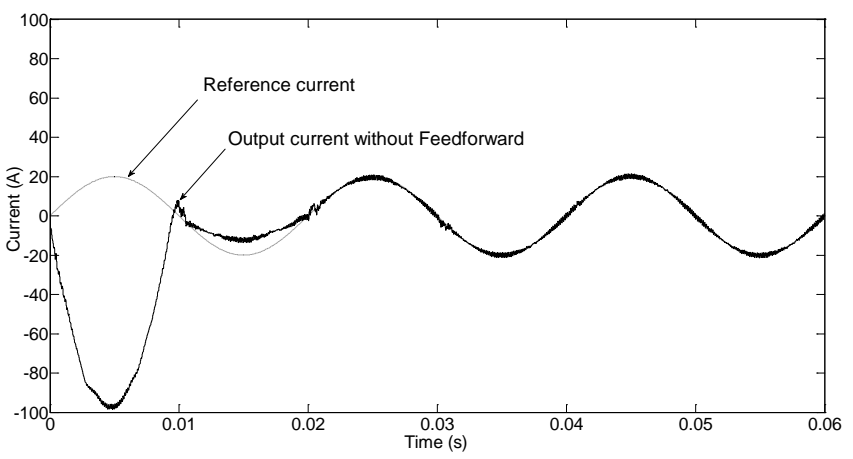

(a) Simulated output current with RC but without the feedforward 


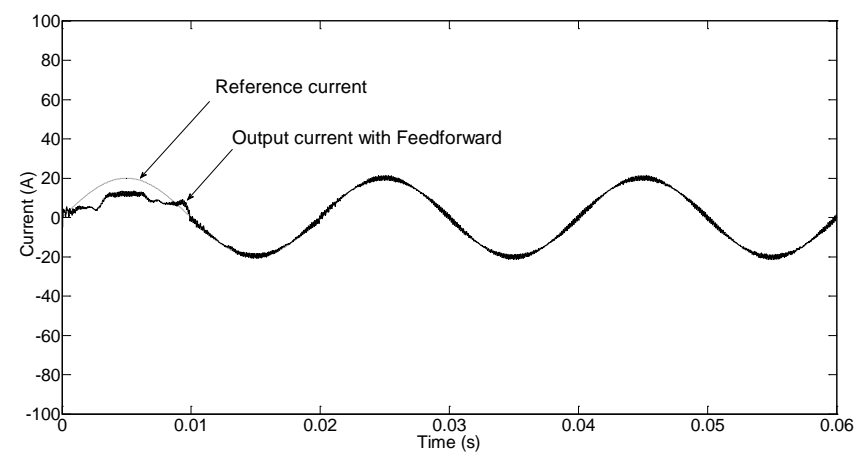

(b) Simulated output current with RC and feedforward

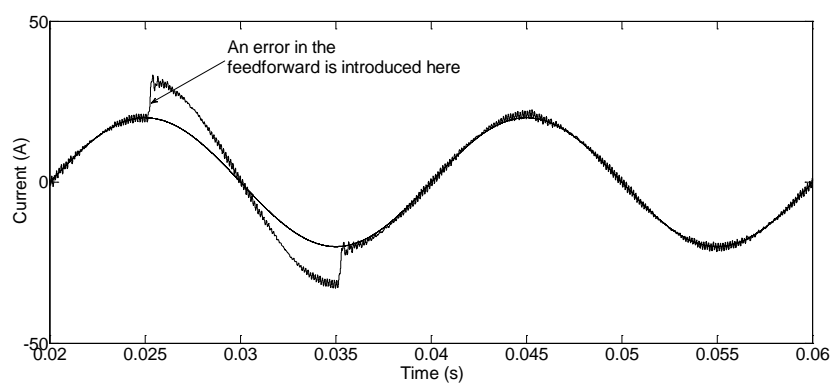

(c) Output current with sudden change in the feedforward

Fig. 7. Simulated output current.

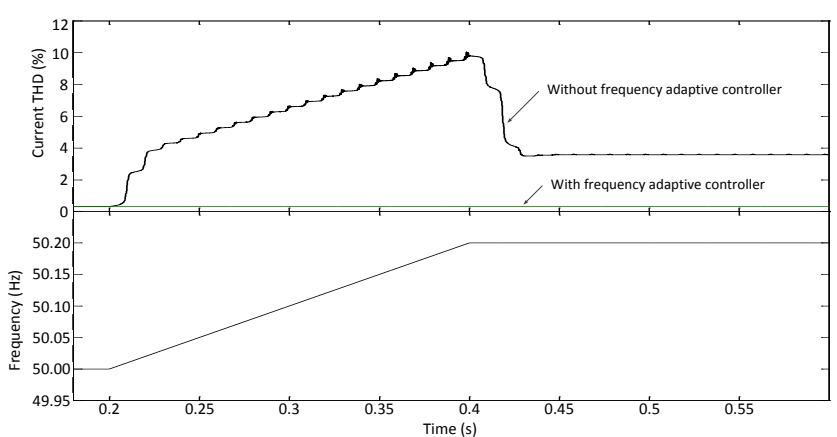

Fig. 8. Output current THD (Grid frequency started to change from $50 \mathrm{~Hz}$ to $50.2 \mathrm{~Hz}$ at $\mathrm{t}=0.2 \mathrm{~s}$ with a slope of $1 \mathrm{~Hz} / \mathrm{sec}$ )

\section{PRACTICAL IMPLEMENTATION AND EXPERIMENTAL RESUlTS}

The proposed controller was tested experimentally with the grid-connected inverter described in Fig. 1 and Table I. The control parameters were listed in Table II. The controller was implemented using a Texas Instrument TMS320F2812 32-bit fixed point DSP. The control algorithm was written in C programming language and compiled using Texas-Instrument Code Composer V5.3 tool. 
The three-phase reference sine waves were generated internally by the DSP using lookup tables of $n=320$ samples. The sine waves amplitude is set externally (by a setting in the user interface) and sent via Controller Area Network (CAN)-bus. The input DC is regulated by an external boost circuit to $700 \mathrm{~V}$ dc so current can be injected into the 230Vrms grid.

The RC controller has been realized by programming as follow: from (2), the discreet transfer function the relates the RC output $Y(z)$ to the RC input $E(z)$ is given by

$$
\frac{Y(z)}{E(z)}=-\frac{K_{R} z^{m} Q(z) z^{-n / 2}}{1+Q(z) z^{-n / 2}}
$$

Substituting (7) in (18) and rearranging gives

$$
Y(z)=X(z)\left(\alpha_{1} z^{1+m-n / 2}+\alpha_{0} z^{m-n / 2}+\alpha_{1} z^{m-n / 2-1}\right),
$$

where $X(z)$ is given by

$$
X(z)=-z^{-m} Y(z)-K_{R} E(z)
$$

Equations (19) and (20) represent the indirect (standard) realization of digital controllers [33] and they are represented by the block diagram in Fig. 9. The RC controller is implemented in software by creating 3 arrays $x(i)$ (one per phase) each is 160 (320/2) entries long. At the discrete time $i$, the RC output $y(i)$ is calculated using the difference equation (21) and the array entry $x(i)$ is filled using the difference equation (22)

$$
\begin{gathered}
y(i)=\alpha_{1} x(i+1+m-n / 2)+\alpha_{0} x(i+m-n / 2)+\alpha_{1} x(i+m-n / 2-1), \\
x(i)=-y(i-m)-K_{R} e(i),
\end{gathered}
$$

where $e(i)$ is the current error at discrete time $i$.

In order to implement the proposed frequency adaptive control, a high precision measurement of the grid voltage frequency is required. The grid voltage signal is sensed and the Positive-Going Zero Crossing (PGZC) is detected. The grid voltage fundamental period $T_{g}$ is measured by calculating the number of samples between consecutive PGZC. In order to increase the measurement accuracy, the PGZC is detected every 15 fundamental cycles. In this way, the measurement error is reduced to $T_{s} / 15$ (compared to $T_{s}$ of the PGZC is detected every cycle). For the nominal sampling frequency of $16 \mathrm{kHz}$, the measurement error is only $\pm 62.5 \mu \mathrm{s} / 15= \pm 4.16 \mu$ s, which is equivalent to $\pm 0.01 \mathrm{~Hz}$. 
Fig. 10a shows the output current when the RC is de-activated. The demand current is set to 15A (rms). The current THD is measured to be $13.0 \%$. Fig. 10b shows the output current but when the RC is activated. The current THD is measured to be only $1.1 \%$. In the system reported in [3] (which is similar to this one but without RC and $K(z)$ is a phase lag), such low THD was only achievable when the output current equals the rated current, i.e., 140A (rms). The RMS of the 50Hz fundamental current in Fig. 10a is 13A which is slightly less than the demanded RMS of 15A due to the deficiency of the pure proportional controller used. However, The RMS of the $50 \mathrm{~Hz}$ fundamental current in Fig. 10b is $15 \mathrm{~A}$ which means it follows very well the demanded current.

Fig. 10c shows the measured output current harmonics with and without the RC. The feedforward loop was activated all the time to avoid damaging high currents (see the simulation results in Fig. 7a). Fig. 10d shows the moment when the inverter connects to the grid. Before the main contactor closed, the inverter was producing output voltage that was matching exactly the grid voltage thanks to the feedforward loop of the nominal voltage of the fundamental component. This guaranteed smooth connection transient and low current when the contactor closed. To test the effect of the feedforward loop on the output current, an error of 35V is suddenly introduced in the feedforward loop as shown in Fig 10e. This causes immediate change in the output current. The RC corrects for this error but it takes half fundamental cycle to converge. The experimental result agrees with the simulation result in Fig 7c.

The grid frequency was monitored in the laboratory and the maximum deviation recorded was $\pm 0.1 \mathrm{~Hz}$. The current THD was always maintained below $1.2 \%$. In order to test the adaptive frequency controller to a higher grid frequency deviation, an AC voltage source would need to be used to emulate the grid.

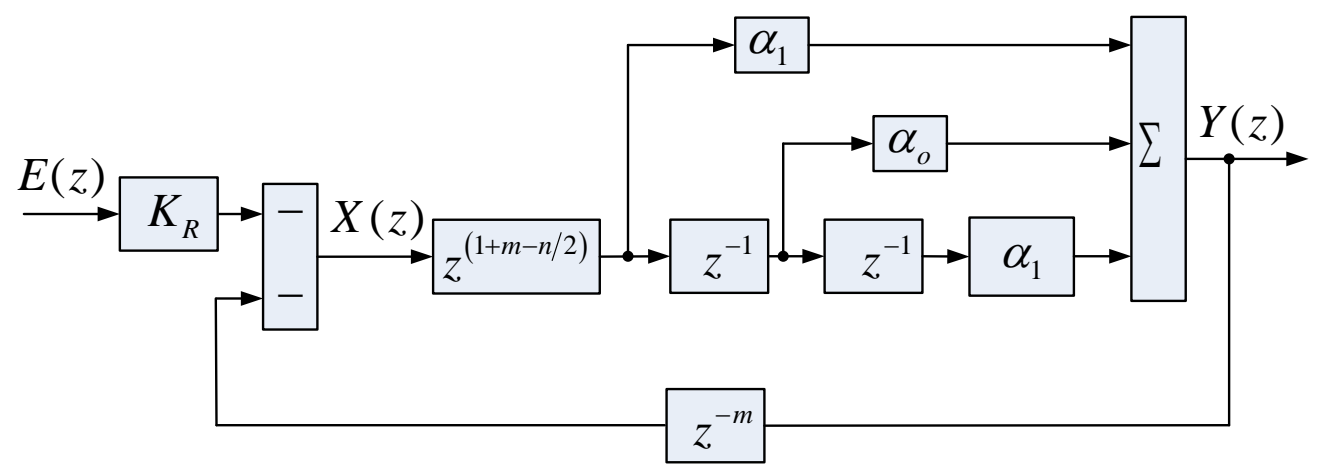

Fig. 9. RC Implementation 


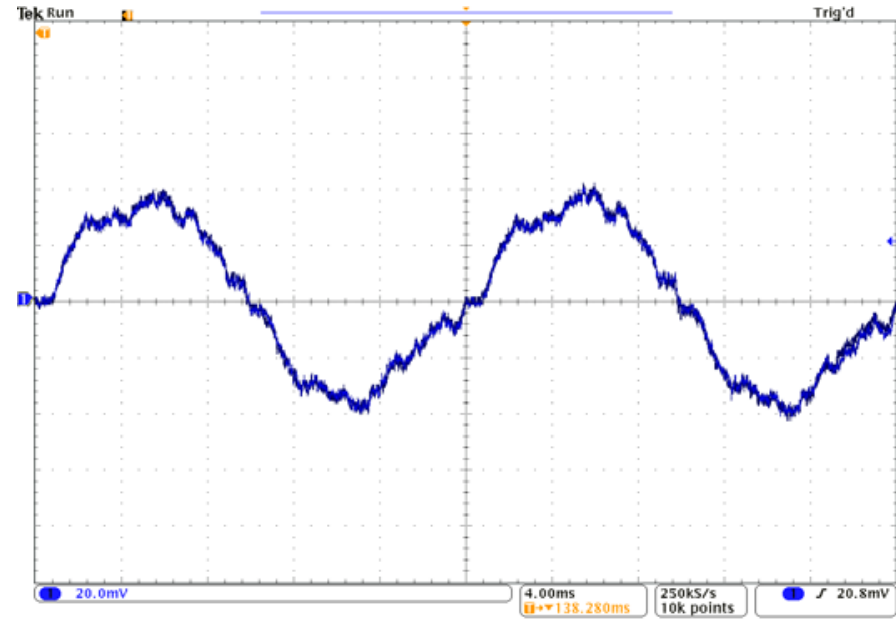

(a) Output current without RC (10A/div)

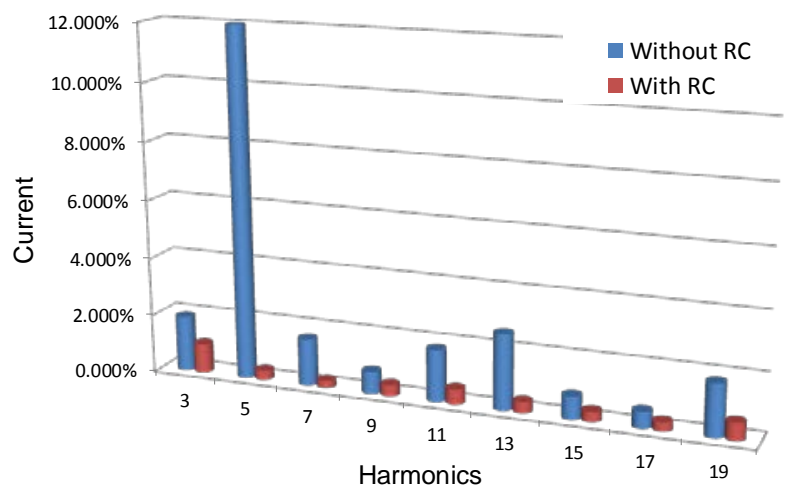

(c) Experimental output current harmonics

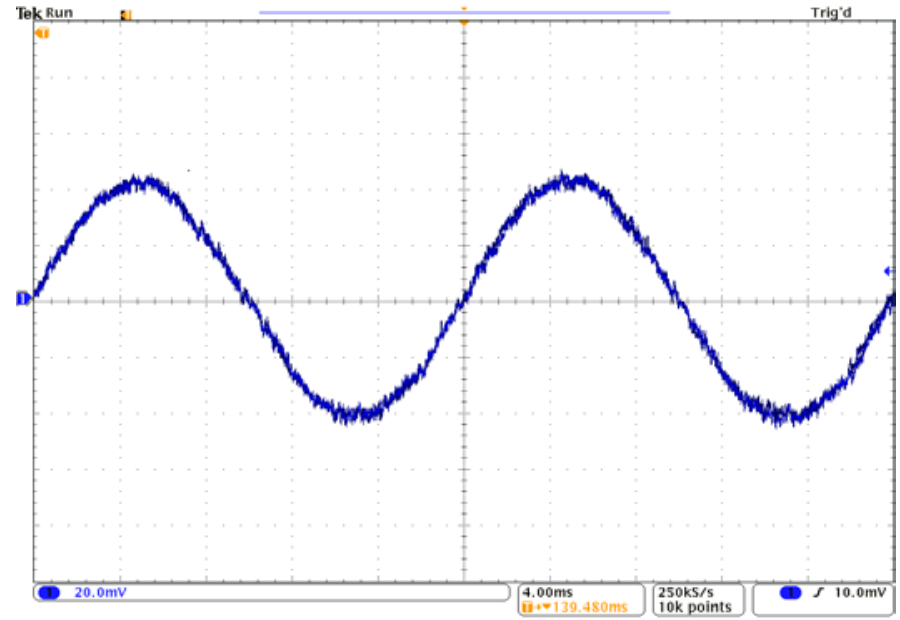

(b) Output current with RC (10A/div)

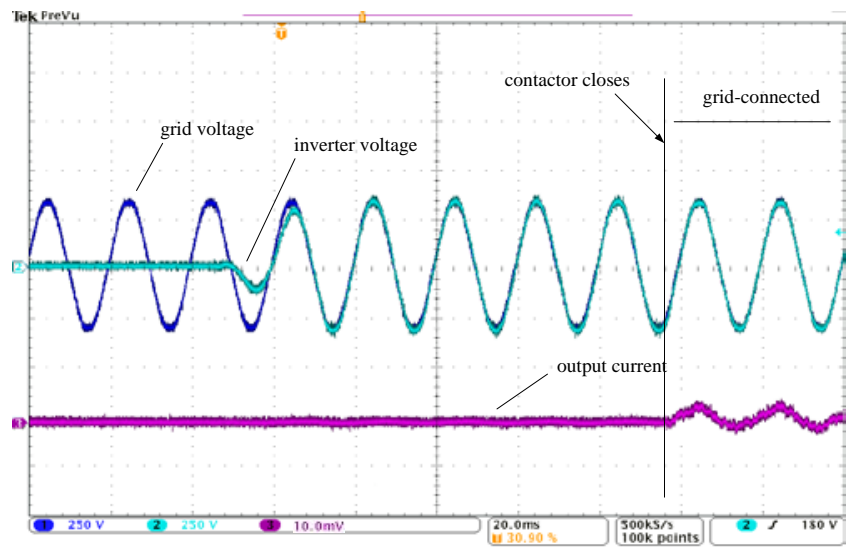

(d) Inverter output voltage before and after connecting to the grid (5A/div)

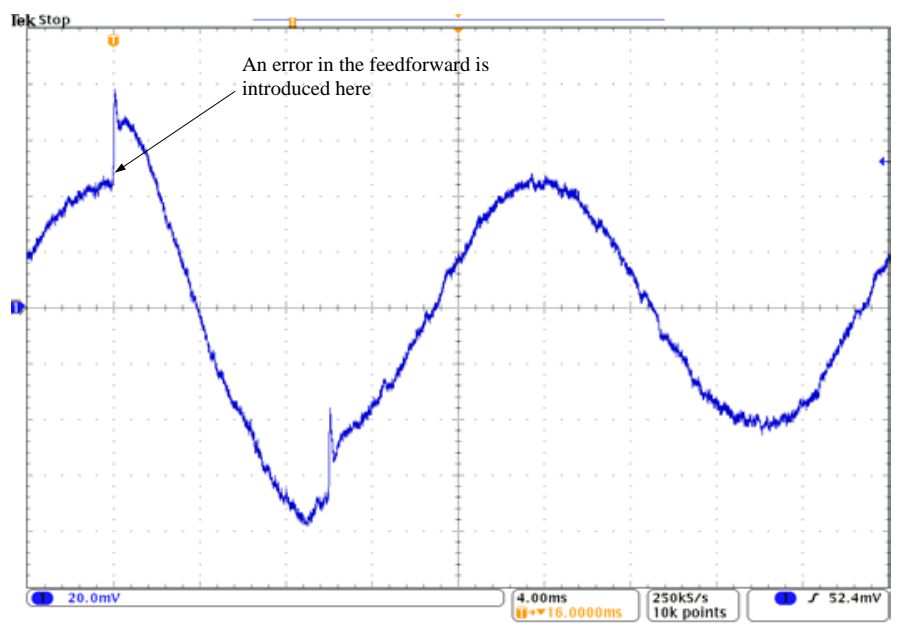

(e) Output current with a sudden change in the feedforrwad (10A/div)

Fig. 10. Practical results 


\section{CONCLUSION}

The design and practical implementation of a frequency adaptive odd-harmonic repetitive controller for a grid-connected inverter was discussed. The adaptive mechanism was found to be very effective in tracking the changes in grid frequency and hence maintaining the effectiveness of the repetitive controller. The proposed mechanism presents a straightforward implementation using a DSP system. The inherent long delay feature of RC has been overcome by implementing a feedforward loop at the fundamental frequency to ensure that the current is bounded during the convergence period of the repetitive controller. The Simulation and experimental results demonstrate the controller's capabilities.

\section{REFERENCES}

[1] Moreno, J. C., Huerta, J. M. E., Gil, R. G. et al.: 'A robust predictive current control for three-phase grid-connected inverters’, IEEE Trans. Ind. Electron., 2009, 56, (6), pp.1993-2004.

[2] Zhao, W., Lu, D. D.C., Agelidis, V. G.: ‘current control of grid-connected boost inverter with zero steady-state error’, IEEE Trans. on Power Electron., 2011, 26, (10), pp. 2825-2834.

[3] Abusara, M. A., Sharkh, S. M.,: 'Digital control of a three-phase grid connected inverter,' Int. J Power Electronics, 2011, 3, (3), pp. 299-319.

[4] Shuitao, Y., Qin, L., Peng, F. Z., et al.: ‘A robust control scheme for grid-connected voltage-source inverters’, IEEE Trans. Ind. Electron., 2011, 58, (1), pp. 202-212.

[5] IEEE1547.1: 'IEEE standard for interconnecting distributed resources with electric power systems', 2003.

[6] IEC1727: 'Characteristic of the utility interface for photovoltaic (PV) systems’, 2002.

[7] Teodorescu, R., Blaabjerg, F., Liserre, M., et al.: 'Proportional-resonant controllers and filters for utility-connected voltagesource converters’, IEE Proceedings on Electric Power Applications, 2006, 153, pp. 750-762.

[8] Hwang, J. G., Lehn, P. W., Winkelnkemper, M.: 'A generalized class of stationary frame-current controllers for utilityconnected AC-DC converters’, IEEE Trans. on Power Delivery, 2010, 25, pp. 2742-2751.

[9] Eren, S., Bakhshai, A., Jain, P.: 'Control of three-phase voltage source inverter for renewable energy applications’, IEEE International Telecommunications Energy Conference, Amsterdam, 2011.

[10] Shen, G., Zhu, X., Zhang, J., et al.: ‘A new feedback method for PR current control of LCL-filter based utility-connected inverter', IEEE Trans. Ind Electron., 2010, 57, (6), pp. 2033-2041. 
[11] Liserre, M., Teodorescu, R., Blaabjerg, F.: 'Multiple harmonics control for three phase utility converter systems with the use of PI-RES current controller in a rotating frame’, IEEE Trans. Power Electron. , 2006, 21, (3), pp. 836-841.

[12] Timbus, A., Ciobotaru, M., Teodorescu, R., et al.: 'Adaptive resonant controller for grid-connected converters in distributed power generation systems', Proc. 21st Annu. IEEE Appl. Power Electron.Conf., 2006, pp. 1601-1606.

[13] Gonzalez-Espin, Garcera, F., G., Patrao, I., et al.: ‘An adaptive control system for three-phase photovoltaic inverters working in a polluted and variable frequency electric grid’, IEEE Trans. Power Electron, 2012, 27, (10), pp. 4248-4261.

[14] Chen, S.L., Hsieh, T. H.: 'Repetitive Control Design and Implementation for Linear Motor Machine Tool', International Journal of Machine Tools and Manufacture, 2007, 47, pp. 1807-16.

[15] Alexandrov, V., van Albada, G., Sloot, P., et al.: 'A Novel Method of Adaptive Repetitive Control for Optical Disk Drivers', The $6^{\text {th }}$ International Conference on Computational Science, Reading, U.K. May 28-31, 2006,

[16] Tinone, H., Aoshima N.: 'Parameter identification of robot arm with repetitive control', International Journal of Control, 1996, 63, pp. $225-238$.

[17] Jiang, S., Cao, D., Li, Y., Liu, J., et al.: 'Low-THD, Fast-transient, and cost-effective synchronous-frame repetitive controller for three-phase UPS inverters’, IEEE Trans. Power Electron, 2012, 27, pp. 2994-3005.

[18] Escobar, G., Valdez, A. A., Leyva-Ramos, J., et al.: 'Repetitive based controller for a UPS inverter to compensate unbalance and harmonic distortion’, IEEE Trans. Ind. Electron., 2007, 54, (1), pp. 504-510.

[19] Grĩn'o, R., Cardoner, R., Costa-Castell'o, R., et al.: ‘Digital repetitive control of a three-phase four-wire shunt active filter’, IEEE Trans. Ind. Electron., 2007, 54, (3), 1495-1503.

[20] Castello, R., Grino, R., Fossas, E.: ‘Odd-harmonic digital repetitive control of a single-phase current active filter’, IEEE Trans. Power Electron., 2004, 19, (4), pp. 1060-1068.

[21] Mattavelli, P., Marafao, F. P.: 'Repetitive-based control for selective harmonic compensation in active power filters', IEEE Trans. Ind. Electron., 2004,51, (5), 1018-1024.

[22] Escobar, G., Leyva-Ramos, J., Mart'inez, P. R., et al.: 'A repetitive-based controller for the boost converter to compensate the harmonic distortion of the output voltage', IEEE Trans. Control Syst. Technol., 2005, 13, (3), pp. 500-508.

[23] Escobar, G., Hern'andez-G'omez, M., Mart'ınez, P. R., et al.: 'A repetitive-based controller for a power factor precompensator', IEEE Trans. Circuits Syst. I, 2007, 54, (9), pp. 1968-1976.

[24] Hornik, T., Zhong, Q. C.: 'Hळ repetitive voltage control of grid connected inverters with a frequency adaptive mechanism', IET Power Electron. , 2010, 3, (6), pp. 925-935. 
[25] Hornik, T., Zhong, Q.C.: 'A current-control strategy for voltage-source inverters in microgrid based on H-infinity and repetitive control’, IEEE Trans. Power Electron., 2011 26, pp. 943-952.

[26] Lu, W., Zhou, K., Yang, Y.: ‘A general internal model principle based control scheme for CVCF PWM converters’, $2^{\text {nd }}$ IEEE International Symposium on Power Electronics for Distributed Generation Systems (PEDG), Hefei China, 2010.

[27]Zhou, K., Wang, D.: 'Digital repetitive learning controller for three phase CVCF PWM inverter', IEEE Trans. Ind. Electron., 2001, 48, (4), pp. 820-830.

[28]Zhang, K., Kang, Y., Xiong, J., et al.: 'Direct repetitive control of SPWM inverters for UPS purpose’, IEEE Trans. Power Electron., 2003, 18, (3), pp. 784-792.

[29]Deng, H., Oruganti, R., Srinivasan, D.: ‘Analysis and design of iterative learning control strategies for UPS inverters’, IEEE Trans. Ind. Electron., 2007, 54, (3), pp. 1739-1751.

[30] Costa-Castello, R., Grino, R., Fossas, E.: 'Odd-harmonic digital repetitive control of a single-phase current active filter', IEEE Trans. Power Electron., 2004, 19, (4), pp. 1060-1068.

[31]Zhou, K., Low, K., Wang, D., et al.: ‘Zerophase odd-harmonic repetitive controller for a single-phase PWM inverter’, IEEE Trans. Power Electron., 2006, 21, (1), pp. 193-201.

[32] Michels, H. P., Grundling, H.: 'Design of plug-in repetitive controllers for single-phase PWM inverters', 39th IAS Annual Meeting: Industry Applications Conference, 2004.

[33] Ogata K.: 'Discrete-time control systems’, (Prentice-Hall, 1995). 